\title{
Evolution of epithelial morphogenesis: phenotypic integration across multiple levels of biological organization
}

\author{
Thorsten Horn, Maarten Hilbrant and Kristen A. Panfilio* \\ Institute for Developmental Biology, University of Cologne, Cologne, Germany
}

Morphogenesis involves the dynamic reorganization of cell and tissue shapes to create the three-dimensional body. Intriguingly, different species have evolved different morphogenetic processes to achieve the same general outcomes during embryonic development. How are meaningful comparisons between species made, and where do the differences lie? In this Perspective, we argue that examining the evolution of embryonic morphogenesis requires the simultaneous consideration of different levels

\section{OPEN ACCESS}

Edited by:

Sylvain Marcellini,

University of Concepcion, Chile

Reviewed by:

David Q. Matus,

Stony Brook University, USA

Stefanie D. Hueber,

University of Konstanz, Germany

*Correspondence:

Kristen A. Panfilio,

Institute for Developmental Biology, University of Cologne, Zülpicher Str.

47b, 50674 Cologne, Germany kristen.panfilio@alum.swarthmore.edu

Specialty section:

This article was submitted to Evolutionary and Population Genetics, a section of the journal

Frontiers in Genetics

Received: 30 June 2015 Accepted: 11 September 2015 Published: 29 September 2015

Citation:

Horn T, Hilbrant M and Panfilio KA (2015) Evolution of epithelial

morphogenesis: phenotypic integration across multiple levels

of biological organization

Front. Genet. 6:303.

doi: 10.3389/fgene.2015.00303 of biological organization: (1) genes, (2) cells, (3) tissues, and (4) the entire egg, or other gestational context. To illustrate the importance of integrating these levels, we use the extraembryonic epithelia of insects - a lineage-specific innovation and evolutionary hotspot-as an exemplary case study. We discuss how recent functional data, primarily from RNAi experiments targeting the Hox3/Zen and U-shaped group transcription factors, provide insights into developmental processes at all four levels. Comparisons of these data from several species both challenge and inform our understanding of homology, in assessing how the process of epithelial morphogenesis has itself evolved.

Keywords: epithelial morphogenesis, evolution of development, insects, extraembryonic tissues, Hox3/zen, Tribolium castaneum, Megaselia abdita, Oncopeltus fasciatus

\section{Introduction}

In the rapidly developing fruit fly Drosophila melanogaster, the predominant insect model for developmental genetics, embryonic morphogenesis occurs largely after cell fates are determined. Indeed, there is extensive literature on Drosophila early tissue patterning, including axis specification and segmentation, preceding morphogenesis. Perhaps as a result of our profound knowledge in Drosophila, many evolutionary developmental (evo-devo) studies in arthropods take a gene-centered approach and focus on early patterning, as early fate specification is often a powerful signal for comparisons of species that are separated by long periods of evolutionary time (e.g., Peel et al., 2005; Sachs et al., 2015).

In this Perspective article, however, we highlight the importance of studying the morphogenetic movements that occur during animal development and of integrating multiple levels of biological organization when making interspecific comparisons. For doing so, we distinguish between four increasingly inclusive levels of biological organization. (1) Genetic regulation of development comprises information about the specific genes and their protein products that are involved in transcriptional control, signaling cascades, and the molecular basis of cytoskeletal structure and remodeling. (2) Individual cells differentiate to acquire a particular identity, including 
the transcriptional state as well as cell shape and structure. (3) More broadly, cells coordinate with their neighbors within tissues. In epithelial tissues for example, cells retain contact with their neighbors via adherens junctions, such that cell shape changes affect the entire tissue's geometry. (4) Finally, the egg is a global system, where tissue integrity and inter-tissue adhesion need to be precisely controlled during morphogenesis to achieve the final form.

Overall, integration of different biological levels is as much a conceptual framework for understanding the physical context of a given gene's role in a developmental process as for interpreting how morphogenesis has evolved. To illustrate this, here we discuss recent advances in the study of extraembryonic (EE) development in a range of insect model species. We show that the insect EE epithelia provide a case study with a particularly rich evolutionary history, making them well suited to assessing the evolution of morphogenesis.

\section{Development and Evolution of the Insect Extraembryonic Membranes}

Many arthropod eggs include an EE tissue component, but in the insects this feature has become a specific structural innovation (reviewed in Panfilio, 2008). At the base of the winged insect lineage, the EE epithelial membranes evolved to form discrete compartments within the egg. In most winged insects, the serosa lines the eggshell, providing the outermost cellular layer and enclosing all other contents, including the yolk. The amnion, analogous to its namesake in vertebrates, forms a fluid-filled cavity ventral to the embryo, retaining a connection to the embryo along the latter's dorsal margin (Figure 1: "most insects" schematic).

The ability of EE membranes to form these compartments early in development has allowed the insects to exploit diverse ecological niches, largely due to the manifold functions of the serosa as a protective outer layer that buffers the embryo against environmental fluctuations and assaults. Recent work has shown that serosal cuticle secretion correlates with the acquisition of desiccation resistance, and that the cuticle itself provides mechanical support to the egg (Rezende et al., 2008; Jacobs et al., 2013; Panfilio et al., 2013). At the same time, recent experimental evidence demonstrates the long hypothesized ability of the serosa to protect the embryo after wounding and pathogen infection via upregulation of the innate immune system (Chen et al., 2000; Jacobs et al., 2014). Furthermore, the serosa's ultrastructure is consistent with physiological roles in water and solute processing, and it has acquired additional mechanical and physiological functions during hatching and early larval life in species with oviposition sites within plant and animal tissues (citations in Panfilio, 2008).

The functional importance of the amnion remains far more enigmatic, despite early recognition of the potential value of an insect amniotic cavity (Zeh et al., 1989). Indeed, the amniotic cavity has been lost independently during the evolution of apocritan wasps and cyclorrhaphan flies (Fleig and Sander, 1988; Rafiqi et al., 2008), with the amniotic epithelium confined to a dorsal yolk cover (Figure 1: schematic for Megaselia). More extremely, in Drosophila melanogaster the serosa and amnion are conflated into a single, dorsal amnioserosa, dispensing with EE compartments entirely (Figure 1: schematic for Drosophila), and some Drosophila species have decanalized development to the point where amnioserosal formation is variable, but still essential (Gavin-Smyth et al., 2013; Panfilio and Roth, 2013).

Using the evolution and development of insect EE membranes as a case study, in the next sections we discuss how the different levels of biological organization are interconnected. We show that, for example, changes at the gene level can induce dramatic changes in cell and tissue behavior that differ between species, even if the consequences at the whole egg level are similar. On the other hand, similar morphogenetic movements can be achieved by quite different mechanisms on the cellular and tissue organizational levels.

\section{Linking zen and U-shaped Genes to Changes at the Egg Level}

Extraembryonic tissue evolution is tightly linked to evolution of the Hox3/Zen transcription factor (Figure 1). The evolutionary origin of strictly EE expression of this gene coincides with the origin of complete EE compartments (Hughes et al., 2004; Panfilio et al., 2006). Hox genes are generally highly conserved in relative genomic position, protein sequence, copy number, and function in anterior-posterior patterning (Krumlauf, 1992; Cook et al., 2001). In contrast, arthropod Hox3 orthologs are prone to duplication and marked sequence divergence, particularly the insect orthologs (known as "zen," after the original Drosophila mutants), with independent instances of duplication in beetles, flies, and lepidopterans (Pultz et al., 1988; Brown et al., 2002; Panfilio et al., 2006; Panfilio and Akam, 2007; Chai et al., 2008; Rafiqi, 2008; Ferguson et al., 2014). Interestingly, in the red flour beetle, Tribolium castaneum, the two zen paralogs have different functions (van der Zee et al., 2005), and these will be discussed in turn.

In all holometabolous insects studied so far zen, or Tc-zen1 in Tribolium, has a conserved function in EE tissue specification (Figure 1: orange diamonds). However, while loss of function mutation in Drosophila is lethal (Wakimoto et al., 1984), the scuttle fly Megaselia abdita and Tribolium can survive after RNA interference (RNAi) knockdown (van der Zee et al., 2005; Rafiqi et al., 2008; Panfilio et al., 2013). Examining why the endstage phenotypes differ after loss of a conserved gene's function provides a good example for the integration of the different levels of biological organization.

Key to understanding the different phenotypic outcomes of disrupting zen/zen 1 is the evolutionary change in EE membrane complement between these species. The single amnioserosa of Drosophila exhibits features of both early serosa and late amnion (reviewed in Schmidt-Ott et al., 2010), and specification of the entire EE domain is under the control of Dm-zen (the paralog $D m-z 2$ is not essential during embryogenesis, Pultz et al., 1988). In the less derived situation in Megaselia and Tribolium, Ma-zen/Tczen 1 only specifies the serosa (van der Zee et al., 2005; Rafiqi et al., 2008). The phenotypic outcome after zen knockdown could then be explained by loss of all EE tissue identity in Drosophila, while Megaselia and Tribolium retain an amnion. 


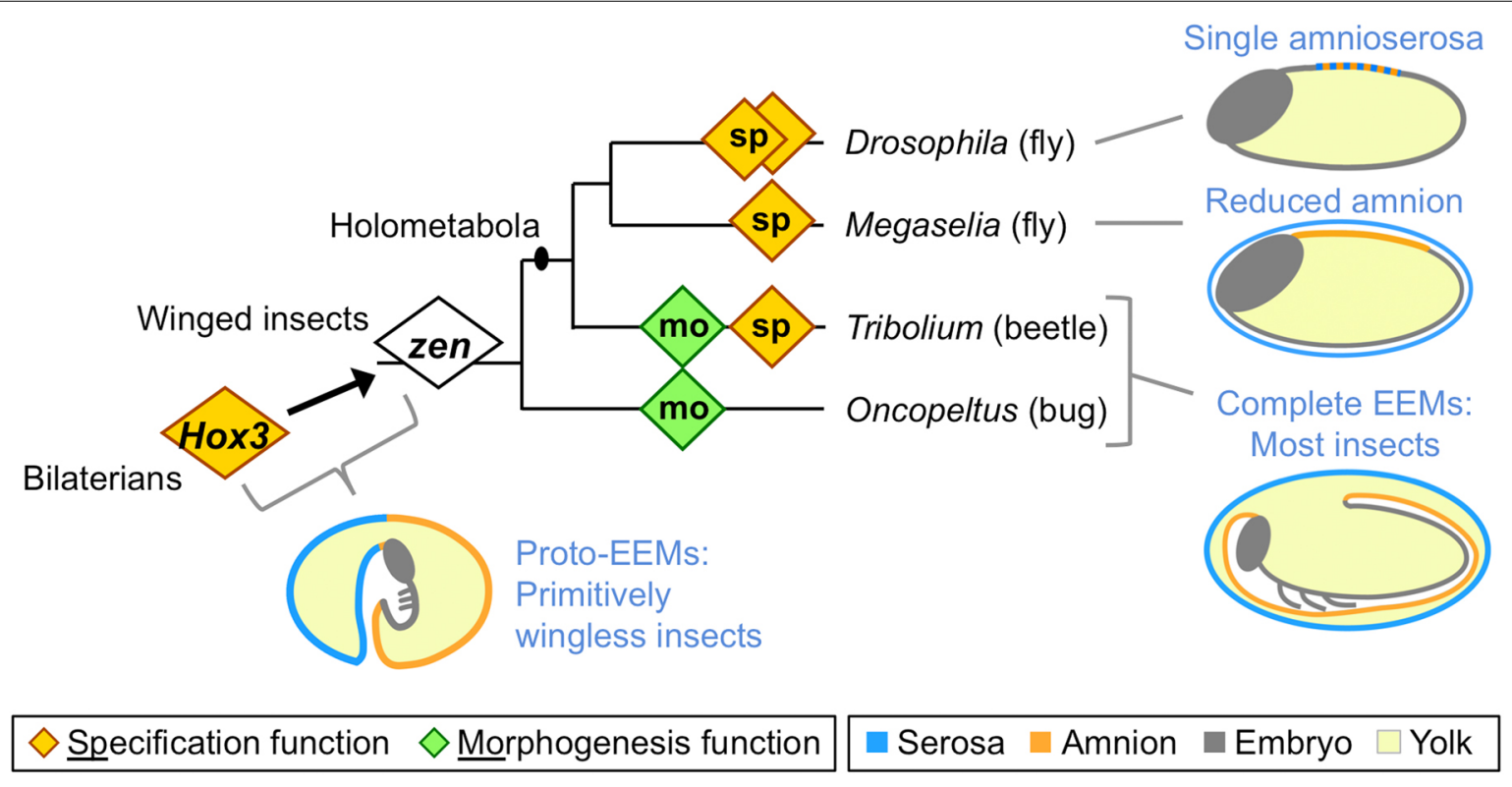

FIGURE 1 | Evolution of extraembryonic membranes (EEMs) and zen gene function in insects. This phylogeny shows species for which functional data on the homeodomain transcription factor Zen are available, and which are discussed here. Diamonds represent individual zen genes (two each in Drosophila and Tribolium), with either a late morphogenetic function (green) or an early specification function (orange). Non-insect Hox3 orthologs also have a specification function, albeit within embryonic rather than extraembryonic tissue. Note that within the fly lineage the highly divergent bicoid paralog has been omitted for clarity (for recent work on this, see Klomp et al., 2015). Schematics show evolutionary stages of EEM acquisition and secondary reduction as inferred from extant species (blue text; color coding is indicated in the legend). Here, "complete" refers to the formation of discrete, closed compartments within the egg, namely the outer serosal sac and the inner amniotic cavity. The illustration of EEM organization in primitively wingless insects is modified from (Panfilio, 2008), with the corresponding author's consent.

However, on closer inspection the similarity in gene function, residual EE tissue complement, and end-stage phenotypic outcome in Megaselia and Tribolium is rather surprising if we consider the difference in EE membrane configuration (Figure 1: schematics). In both species, we observe a respecification from serosal to amniotic fate and in both species it is important to have a tissue covering the yolk dorsally during the dorsal closure stage. However, the underlying wild type configurations are different. In Megaselia, the amnion provides a persistent dorsal yolk cover, and its overall shape, size, and dorsal position are not changed dramatically by the $M a-z e n^{R N A i}$ fate shift (Rafiqi et al., 2008). In contrast, in Tribolium the dorsal side of the egg is first covered by the serosa and only later in wild type development is the amnion pulled dorsally when the serosa contracts (Panfilio et al., 2013). How can Tribolium then survive without a serosa? Here, Tc-zen $1^{R N A i}$ not only produces a persistently dorsal amniotic region due to respecification (van der Zee et al., 2005), but also reveals novel cellular and tissue properties of the entire amnion in late development as it takes over the role of the serosa in providing a dorsal cover (Panfilio et al., 2013). Hence, the survival of $M a-z e n^{R N A i}$ embryos is rather due to the dispensability of the serosa for dorsal closure, while in Tribolium developmental regulation-that is, compensation via plasticity of the amnion-enables survival after Tc-zen $1^{R N A i}$. Thus, conserved, early gene functions can feed into different developmental routes, depending on tissue configuration and morphogenetic properties.

At the same time, other genes with EE roles have undergone changes in their particular function and in their interaction partners during insect evolution. One example is the T-box transcription factor Dorsocross (Doc), a member of the Ushaped gene family (Frank and Rushlow, 1996; Reim et al., 2003). In Drosophila, Dm-Doc is necessary for the maintenance of the amnioserosa toward the end of germband extension, when Zen protein disappears (Reim et al., 2003, and references therein). In contrast, $T c$-Doc has multiple roles in Tribolium EE morphogenesis, but no role in maintaining either EE tissue $(\mathrm{TH}$, KAP unpublished observation). There is some evidence that $\mathrm{Ma}$ Doc has a maintenance function in the Megaselia serosa (Rafiqi et al., 2008), but the end stage RNAi phenotype would also be consistent with an early morphogenetic role, as in Tribolium.

Consistent with this difference in the EE role of Doc, the molecular context of its function also differs between species. Drosophila Doc expression requires simultaneous inputs from Dm-Zen and Dm-Dpp (Reim et al., 2003). In contrast, in Tribolium these inputs are temporally and spatially distinct, and subsequent Dpp signaling is itself locally dependent on $T c-D o c$ (TH, KAP unpublished observation), a feature not known from Drosophila. Another example is Doc's relation to hindsight (hnt), another U-shaped gene. In Drosophila, Dm-hnt is downstream of Dm-Doc and therefore shows a similar knockdown phenotype. In Tribolium, both genes also show a similar knockdown phenotype to one another, but they seem not to influence each other's expression (TH, KAP unpublished observation).

Finally, Dm-Doc performs multiple functions within the body proper (Hamaguchi et al., 2012; Sui et al., 2012), such as for heart development (Reim and Frasch, 2005), that are not observed in Tribolium (Nunes da Fonseca et al., 2010). 
Interestingly, one of these functions, bending of the Drosophila wing imaginal disc, directly links the transcription factor Dm-Doc to cellular and epithelial rearrangements (Sui et al., 2012). Here, Dm-Doc promotes intracellular microtubule web redistribution and degradation of the extracellular matrix through Matrix metalloproteinase. It remains to be seen if similar mechanisms are also employed downstream of Doc in EE morphogenesis across species.

In summary, disruption of zen, a gene with a conserved function in specification of the serosa, leads to lethality, compensation by the amnion, or simply loss of the serosa with no severe consequences for development, depending on the tissue topography of the species under investigation. Moreover, there are large differences in gene knockdown phenotypes, overall gene functions, and specific interaction partners between orthologous genes in different species, and these differences can only be understood if all other biological levels, from cells to the egg system, are taken into account.

In the next section we shift the focus from the genes themselves to tissue organization and function, again highlighting differences between species at different levels of biological organization.

\section{Linking Cellular, Tissue, and Egg System Levels}

In late embryogenesis, it is essential that insect EE tissue actively withdraws in a precise way to mediate dorsal closure, whereby the embryonic epidermis seals at the dorsal midline and EE tissue degenerates within the yolk. Indeed, amnioserosa-epidermal tissue coordination during Drosophila dorsal closure has been extensively studied over the last 15 years (e.g., Jacinto et al., 2000; Kiehart et al., 2000; Solon et al., 2009; Lada et al., 2012; Wells et al., 2014). While differences in tissue organization are expected between dorsal closure involving an amnioserosa and dorsal closure involving a serosa and amnion, we also find differences between species with both EE membranes (Figure 1: "most insects" schematic). To illustrate this point, here we compare late EE morphogenesis between Tribolium and the hemimetabolous milkweed bug, Oncopeltus fasciatus, charting a sequence of similarities and differences as morphogenesis proceeds.

Firstly, rupture of the EE tissues over the embryo's head produces an opening through which the embryo passively emerges. In Oncopeltus, preparation for EE rupture within this specialized region involves apoptosis of the amniotic cells subjacent to the serosa, thinning the region to a single EE epithelium, while at the border of this region the amnion adheres strongly to the serosa (Panfilio and Roth, 2010). As this epithelial remodeling occurs locally, the entire egg system is subtly reorganized to ensure that the specialized EE region is centered at the egg pole, which appears to mechanically facilitate rupture via global contractile force exerted by the serosa (Panfilio, 2009; Panfilio and Roth, 2010). In contrast, in Tribolium the opening for EE rupture is not centered at the egg pole but occurs anteriorventrally (Panfilio et al., 2013). Here, precision in determining the site of EE opening involves morphological specialization in a cap of amniotic cells. Furthermore, preparation for rupture in Tribolium involves the formation of an amnion-serosa epithelial bilayer over most of the amnion's surface area (Koelzer et al., 2015), not just the narrow ring of amnion-serosa contact seen in Oncopeltus. These differences in local behavior of the amnion and in the amnion-serosa connection are all the more striking given that $O f-z e n$ and $T c-z e n 2$, the second Tribolium paralog, both act extraembryonically to ensure that EE rupture occurs (Figure 1: green diamonds; van der Zee et al., 2005; Panfilio et al., 2006).

In subsequent stages the EE tissues withdraw dorsally, but with the serosa ending up in the tapered dorsal-anterior in Oncopeltus compared to the flat dorsal-medial region in Tribolium (Figures 2A,E). Nonetheless, in both cases the serosa transforms from a squamous to a columnar epithelium and forms a hollow disc known as the dorsal organ (Panfilio, 2009; Panfilio and Roth, 2010; Panfilio et al., 2013; Figures 2B,C,F,G). Thus, cell shape and intra-tissue organization are conserved despite the geometrical difference resulting from the tissues' positions within an anisotropic egg system.

However, as a consequence of the manner in which the amnion-serosa connection was prepared for rupture, the intertissue organization remains fundamentally different at the dorsal organ stage. The Oncopeltus amnion is only connected to the serosa at its margin, and sits on top of the yolk (Figure 2D). While both this attachment point and substrate also apply to the Tribolium amnion, the bilayer organization means that additionally a portion of the amnion has the serosa as a substrate (Figure 2H). As the serosa degenerates, tissue continuity over the yolk surface is essential for successful dorsal closure (Panfilio et al., 2013). The planar (lateral-lateral) nature of amnion-serosa attachment in Oncopeltus allows the serosa to efficiently pinch off and draw the edges of the amnion together above it (Figure 2B). In Tribolium, inter-tissue shearing is required so that the portion of the amnion over the serosa (apical-basal connection) can detach, enabling final serosal internalization (Koelzer et al., 2015).

Altogether, zen-mediated rupture, EE contraction and withdrawal, and the cellular structure of the serosal dorsal organ are shared between Oncopeltus and Tribolium even though the manner of amniotic regionalization (selective apoptosis or morphological alteration) and therefore the nature of the amnion-serosa inter-tissue connection differ.

\section{Conclusions}

In this Perspective, we use morphogenesis of the insect EE epithelia to show how different levels of biological organization can provide apparently contradictory signals as to the degree of evolutionary conservation across species. At first glance, these levels are hierarchically ordered, with increasing complexity toward the whole egg system: genes specify cell types and shapes, cells of similar type form tissues, and different tissues shape the whole egg system morphology. However, any pattern of congruence across levels is possible. For example, zen orthologs are necessary to specify the serosa in holometabolous insects, but the loss of zen function is lethal in some species, while others survive-variously due to serosal dispensability or morphogenetic compensation by the amnion. These phenotypic 
A

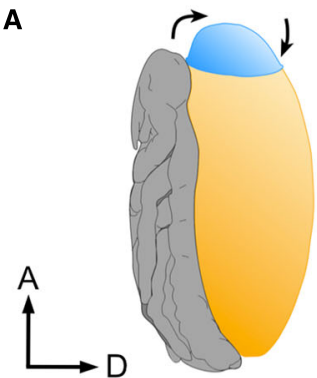

B

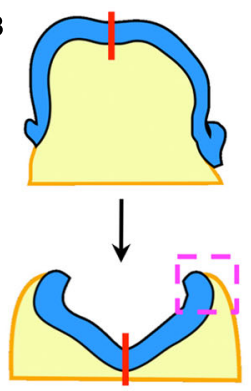

c

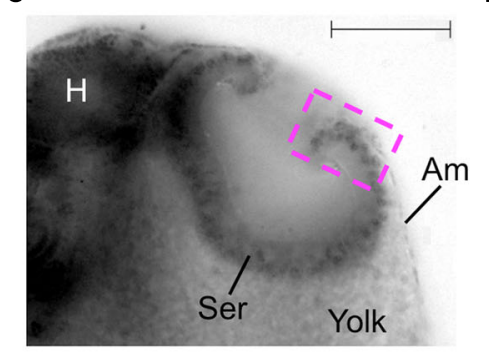

D

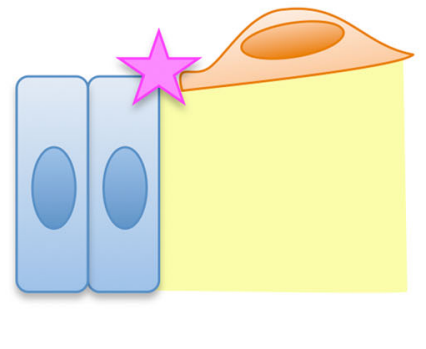

E

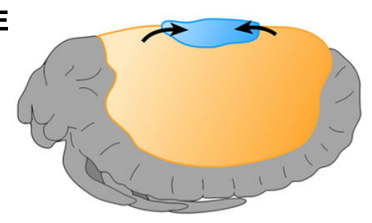

$A \longleftarrow$
F

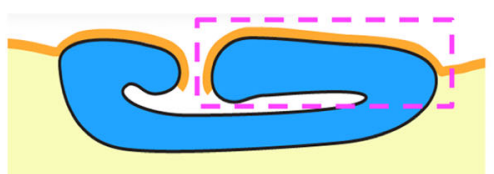

G

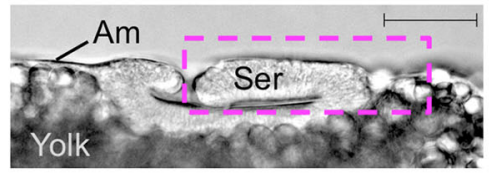

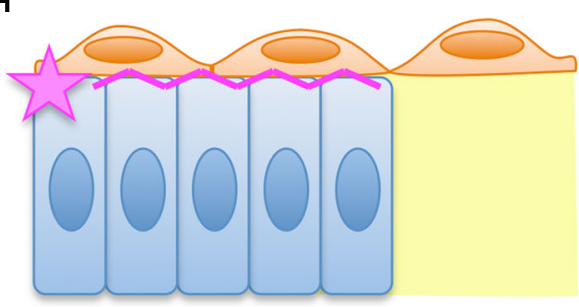

Gerosa $\square$ Amnion $\square$ Embryo $\square$ Yolk $\square$ Inter-tissue attachment

FIGURE 2 | Different tissue organizations achieve the same morphogenetic outcomes. At the dorsal organ stage the serosa compacts into a hollow disc that sinks into the yolk, shown here for Oncopeltus fasciatus (A-D) and Tribolium castaneum (E-H) as representative of hemi- and holometabolous insects, respectively. Although the site of dorsal organ formation differs in relative position and geometry within the global egg system (A,E), in both cases serosal cells become columnar as the tissue everts (B,C,F,G). The process of serosal eversion is shown schematically in $B$, where the red line indicates the center of the serosa during this process. In yet another difference, the nature of amnion-serosa attachment consists of a lateral junction within the plane of the extraembryonic epithelium in Oncopeltus (D: star), while the bilayered arrangement in Tribolium additionally involves basal-basal contact of the two tissues (H: zigzag line). All views are lateral except (B), which is dorsal and omits the embryonic tissue for simplification. Dashed boxes indicate the region of inter-tissue attachment, which is shown schematically at the cellular level in (D) and (H). Micrographs show fixed embryos with a nuclear stain (C) or DIC illumination (D), with scale bars of $100 \mu \mathrm{m}$ and $50 \mu \mathrm{m}$, respectively. Abbreviations: A, anterior; Am, amnion; D, dorsal; H, head; Ser, serosa. Images A,B,C,E are reproduced with minor modification from (Panfilio, 2008, 2009; Panfilio and Roth, 2010; Panfilio et al., 2013), with the corresponding author's consent.

outcomes can be explained by a conserved gene function being embedded in the context of differences in EE tissue complement and topographical configuration across species. In the case of EE epithelial withdrawal in Oncopeltus and Tribolium, the nature of tissue regionalization and inter-tissue attachment differ dramatically even while gene function, intra-tissue structure, and gross morphogenesis are similar. Only the integration of all biological levels can provide the full picture and give insight into the evolution not just of epithelial morphogenesis but of embryogenesis in general, which ultimately depends on cell shape changes and coordinated tissue reorganization.

In the past, these levels have predominantly been studied separately or in limited combinations. In the watershed Heidelberg screen of Drosophila embryonic patterning mutants (Nüsslein-Volhard and Wieschaus, 1980), gene function was linked to final phenotype as determined from larval cuticle preparations, a method still widely employed, especially for large scale screening (Schmitt-Engel et al., 2015). However, even as the initial link between gene and egg system levels is being established, the aim is to refine this information to more precise phenotypic analysis. At this point, a misexpressed gene itself becomes a tool to further explore cell and tissue properties.
With new techniques available, we are increasingly able to investigate multiple levels of biological organization at the same time. For example, gene silencing via RNAi combined with live imaging of fluorescent constructs that afford cell and tissue resolution allows us to visualize the full developmental phenotype resulting from a given genetic manipulation, with Tribolium serving as a particularly amenable comparative model among the insects (Sarrazin et al., 2012; Benton et al., 2013; Panfilio et al., 2013; Koelzer et al., 2014). Also, as pioneered in Drosophila, mechanical manipulations provide a means of circumventing genetic manipulation when examining cell, tissue, and egg system levels (e.g., Ma et al., 2009; Monier et al., 2010; Wells et al., 2014), and clonal analysis approaches test cellular behaviors at tissue boundaries (Külshammer and Uhlirova, 2013). From all of these studies it becomes increasingly clear that the interplay between the levels is rather similar to a regulatory network (as known from gene interactions), including various interactions and feedback loops, than to a hierarchical structure based on increasing complexity.

Having understood the interplay of the developmental levels within a species, we can now start comparing different species and additional levels. For example, a key aspect of epithelial morphogenesis is the structure of boundaries between different 
tissues, where mechanical forces are transmitted and intertissue attachments are made. To what extent are mechanical, geometric properties of tissues and the egg system a better predictor than phylogenetic relatedness of how similar two species' morphogenetic processes will be? Moving beyond the confines of the egg system, an even more integrated view of the phenotype can be extended to the influence of the external environment, as addressed in the growing field of eco-evo-devo (Gilbert and Epel, 2008; Abouheif et al., 2014). Ultimately, as the number of comparative animal models and accessibility of experimental tools increases, so too should the sophistication of our phenotypic understanding of how development has evolved.

\section{References}

Abouheif, E., Favé, M.-J., Ibarrarán-Viniegra, A. S., Lesoway, M. P., Rafiqi, A. M., and Rajakumar, R. (2014). Eco-evo-devo: the time has come. Adv. Exp. Med. Biol. 781, 107-125. doi: 10.1007/978-94-007-7347-9_6

Benton, M. A., Akam, M., and Pavlopoulos, A. (2013). Cell and tissue dynamics during Tribolium castaneum embryogenesis revealed by versatile fluorescence labeling approaches. Development 140, 3210-3220. doi: 10.1242/dev.096271

Brown, S. J., Fellers, J. P., Shippy, T. D., Richardson, E. A., Maxwell, M., Stuart, J. J., et al. (2002). Sequence of the Tribolium castaneum homeotic complex: the region corresponding to the Drosophila melanogaster Antennapedia Complex. Genetics 160, 1067-1074.

Chai, C.-L., Zhang, Z., Huang, F.-F., Wang, X.-Y., Yu, Q.-Y., Liu, B.-B., et al. (2008). A genomewide survey of homeobox genes and identification of novel structure of the Hox cluster in the silkworm, Bombyx mori. Insect. Biochem. Mol. Biol. 38, 1111-1120. doi: 10.1016/j.ibmb.2008.06.008

Chen, G., Handel, K., and Roth, S. (2000). The maternal NF-kappaB/Dorsal gradient of Tribolium castaneum: dynamics of early dorsoventral patterning in a shortgerm beetle. Development 127, 5145-5156.

Cook, C. E., Smith, M. L., Telford, M. J., Bastianello, A., and Akam, M. (2001). Hox genes and the phylogeny of the arthropods. Curr. Biol. 11, 759-763. doi: 10.1016/S0960-9822(01)00222-6

Ferguson, L., Marlétaz, F., Carter, J.-M., Taylor, W. R., Gibbs, M., Breuker, C. J., et al. (2014). Ancient expansion of the Hox cluster in Lepidoptera generated four homeobox genes implicated in extra-embryonic tissue formation. PLoS Genet. 10:e1004698. doi: 10.1371/journal.pgen.1004698

Fleig, R., and Sander, K. (1988). Honeybee morphogenesis: embryonic cell movements that shape the larval body. Development 103, 525-534.

Frank, L. H., and Rushlow, C. (1996). A group of genes required for maintenance of the amnioserosa tissue in Drosophila. Development 122, 1343-1352.

Gavin-Smyth, J., Wang, Y.-C., Butler, I., and Ferguson, E. L. (2013). A genetic network conferring canalization to a bistable patterning system in Drosophila. Curr. Biol. 23, 2296-2302. doi: 10.1016/j.cub.2013.09.055

Gilbert, S. F., and Epel, D. (2008). Ecological Developmental Biology: Integrating Epigenetics, Medicine, and Evolution. Sunderland, MA: Sinauer Associates, Inc.

Hamaguchi, T., Takashima, S., Okamoto, A., Imaoka, M., Okumura, T., and Murakami, R. (2012). Dorsoventral patterning of the Drosophila hindgut is determined by interaction of genes under the control of two independent gene regulatory systems, the dorsal and terminal systems. Mech. Dev. 129, 236-243. doi: 10.1016/j.mod.2012.07.006

Hughes, C. L., Liu, P. Z., and Kaufman, T. C. (2004). Expression patterns of the rogue Hox genes Hox3/zen and fushi tarazu in the apterygote insect Thermobia domestica. Evol. Dev. 6, 393-401. doi: 10.1111/j.1525-142X.2004.04048.x

Jacinto, A., Wood, W., Balayo, T., Turmaine, M., Martinez-Arias, A., and Martin, P. (2000). Dynamic actin-based epithelial adhesion and cell matching during Drosophila dorsal closure. Curr. Biol. 10, 1420-1426. doi: 10.1016/S09609822(00)00796-X

Jacobs, C. G. C., Rezende, G. L., Lamers, G. E. M., and van der Zee, M. (2013). The extraembryonic serosa protects the insect egg against desiccation. Proc. R. Soc. B 280, 20131082. doi: 10.1098/rspb.2013.1082

Jacobs, C. G. C., Spaink, H. P., and Zee, M. V. D. (2014). The extraembryonic serosa is a frontier epithelium providing the insect egg with a full-range innate immune response. eLife 3, e04111. doi: 10.7554/eLife.04111

\section{Author Contributions}

KAP conceived the idea for the manuscript. KAP, TH, and $\mathrm{MH}$ jointly wrote the manuscript.

\section{Acknowledgments}

We thank the German Research Foundation (Deutsche Forschungsgemeinschaft) for financial support for conducting some of the experimental investigations described here and during the preparation of this manuscript (grant PA 2044/1-1 to KAP).

Kiehart, D. P., Galbraith, C. G., Edwards, K. A., Rickolla, W. L., and Montague, R. A. (2000). Multiple forces contribute to cell sheet morphogenesis for dorsal closure in Drosophila. J. Cell Biol. 149, 471-490. doi: 10.1083/jcb.149. 2.471

Klomp, J., Athy, D., Kwan, C. W., Bloch, N. I., Sandmann, T., Lemke, S., et al. (2015). A cysteine-clamp gene drives embryo polarity in the midge Chironomus. Science 348, 1040-1042. doi: 10.1126/science.aaa7105

Koelzer, S., Hilbrant, M., Horn, T., and Panfilio, K. A. (2015). The beetle amnion and serosa functionally interact as apposed epithelia. bioRxiv doi: 10.1101/025247

Koelzer, S., Kölsch, Y., and Panfilio, K. A. (2014). Visualizing late insect embryogenesis: extraembryonic and mesodermal enhancer trap expression in the beetle Tribolium castaneum. PLOS ONE 9:e103967. doi: 10.1371/journal.pone.0103967

Krumlauf, R. (1992). Evolution of the vertebrate Hox homeobox genes. Bioessays 14, 245-252. doi: 10.1002/bies.950140408

Külshammer, E., and Uhlirova, M. (2013). The actin cross-linker Filamin/Cheerio mediates tumor malignancy downstream of JNK signaling. J. Cell Sci. 126, 927-938. doi: 10.1242/jcs.114462

Lada, K., Gorfinkiel, N., and Arias, A. M. (2012). Interactions between the amnioserosa and epidermis revealed by the function of the u-shaped gene. Biol. Open 1, 353-361. doi: 10.1242/bio.2012497

Ma, X., Lynch, H. E., Scully, P. C., and Hutson, M. S. (2009). Probing embryonic tissue mechanics with laser hole drilling. Phys. Biol. 6:036004. doi: 10.1088/14783975/6/3/036004

Monier, B., Pélissier-Monier, A., Brand, A. H., and Sanson, B. (2010). An actomyosin-based barrier inhibits cell mixing at compartmental boundaries in Drosophila embryos. Nat. Cell Biol. 12, 60-65. doi: 10.1038/ncb2005

Nunes da Fonseca, R., van der Zee, M., and Roth, S. (2010). Evolution of extracellular Dpp modulators in insects: the roles of tolloid and twistedgastrulation in dorsoventral patterning of the Tribolium embryo. Dev. Biol. 345, 80-93. doi: 10.1016/j.ydbio.2010.05.019

Nüsslein-Volhard, C., and Wieschaus, E. (1980). Mutations affecting segment number and polarity in Drosophila. Nature 287, 795-801. doi: 10.1038/287795a0

Panfilio, K. A. (2008). Extraembryonic development in insects and the acrobatics of blastokinesis. Dev. Biol. 313, 471-491. doi: 10.1016/j.ydbio.2007.11.004

Panfilio, K. A. (2009). Late extraembryonic development and its zen-RNAi-induced failure in the milkweed bug Oncopeltus fasciatus. Dev. Biol. 333, 297-311. doi: 10.1016/j.ydbio.2009.06.036

Panfilio, K. A., and Akam, M. (2007). A comparison of Hox3 and Zen protein coding sequences in taxa that span the Hox3/zen divergence. Dev. Genes Evol. 217, 323-329. doi: 10.1007/s00427-007-0133-8

Panfilio, K. A., Liu, P. Z., Akam, M., and Kaufman, T. C. (2006). Oncopeltus fasciatus zen is essential for serosal tissue function in katatrepsis. Dev. Biol. 292, 226-243. doi: 10.1016/j.ydbio.2005.12.028

Panfilio, K. A., Oberhofer, G., and Roth, S. (2013). High plasticity in epithelial morphogenesis during insect dorsal closure. Biol. Open 2, 1108-1118. doi: 10.1242/bio.20136072

Panfilio, K. A., and Roth, S. (2010). Epithelial reorganization events during late extraembryonic development in a hemimetabolous insect. Dev. Biol. 340, 100-115. doi: 10.1016/j.ydbio.2009.12.034

Panfilio, K. A., and Roth, S. (2013). Development: getting into the groove, or evolving off the rails? Curr. Biol. 23, R1101-R1103. doi: 10.1016/j.cub.2013.10.073 
Peel, A. D., Chipman, A. D., and Akam, M. (2005). Arthropod segmentation: beyond the Drosophila paradigm. Nat. Rev. Genet. 6, 905-916. doi: $10.1038 / \mathrm{nrg} 1724$

Pultz, M. A., Diederich, R. J., Cribbs, D. L., and Kaufman, T. C. (1988). The proboscipedia locus of the Antennapedia complex: a molecular genetic analysis. Genes Dev. 2, 901-920. doi: 10.1101/gad.2.7.901

Rafiqi, A. M. (2008). Morphological Transitions and the Genetic Basis of the Evolution of Extraembryonic Tissues in Flies WU Plant Sciences, vol. Ph.D. thesis, Wageningen University, Wageningen.

Rafiqi, A. M., Lemke, S., Ferguson, S., Stauber, M., and Schmidt-Ott, U. (2008). Evolutionary origin of the amnioserosa in cyclorrhaphan flies correlates with spatial and temporal expression changes of zen. Proc. Natl Acad. Sci. U.S.A. 105, 234-239. doi: 10.1073/pnas.0709145105

Reim, I., and Frasch, M. (2005). The Dorsocross T-box genes are key components of the regulatory network controlling early cardiogenesis in Drosophila. Development 132, 4911-4925. doi: 10.1242/dev.02077

Reim, I., Lee, H.-H., and Frasch, M. (2003). The T-box encoding Dorsocross genes function in amnioserosa development and the patterning of the dorsolateral germ band downstream of Dpp. Development 130, 3187-3204. doi: 10.1242/dev.00548

Rezende, G. L., Martins, A. J., Gentile, C., Farnesi, L. C., Pelajo-Machado, M., Peixoto, A. A., et al. (2008). Embryonic desiccation resistance in Aedes aegypti: presumptive role of the chitinized serosal cuticle. BMC Dev. Biol. 8:82. doi: 10.1186/1471-213X-8-82

Sachs, L., Chen, Y.-T., Drechsler, A., Lynch, J. A., Panfilio, K. A., Lässig, M., et al. (2015). Dynamic BMP signaling polarized by Toll patterns the dorsoventral axis in a hemimetabolous insect. Elife 4:e05502. doi: 10.7554/eLife. 05502

Sarrazin, A. F., Peel, A. D., and Averof, M. (2012). A segmentation clock with two-segment periodicity in insects. Science 336, 338-341. doi: $10.1126 /$ science. 1218256

Schmidt-Ott, U., Rafiqi, A. M., and Lemke, S. (2010). "Hox3/zen and the evolution of extraembryonic epithelia in insects," in Hox Genes: Studies from the 20th to the 21st Century, vol. 689, ed. J. S. Deutsch (Austin: Landes Bioscience), 133-144.
Schmitt-Engel, C., Schultheis, D., Schwirz, J., Ströhlein, N., Troelenberg, N., Majumdar, U., et al. (2015). The iBeetle large scale RNAi screen reveals novel gene functions for insect development and physiology. Nat. Commun. 6, 7822. doi: $10.1038 /$ ncomms 8822

Solon, J., Kaya-Çopur, A., Colombelli, J., and Brunner, D. (2009). Pulsed forces timed by a ratchet-like mechanism drive directed tissue movement during dorsal closure. Cell 137, 1331-1342. doi: 10.1016/j.cell.2009.03.050

Sui, L., Pflugfelder, G. O., and Shen, J. (2012). The Dorsocross T-box transcription factors promote tissue morphogenesis in the Drosophila wing imaginal disc. Development 139, 2773-2782. doi: 10.1242/dev.079384

van der Zee, M., Berns, N., and Roth, S. (2005). Distinct functions of the Tribolium zerknüllt genes in serosa specification and dorsal closure. Curr. Biol. 15, 624-636. doi: 10.1016/j.cub.2005.02.057

Wakimoto, B. T., Turner, F. R., and Kaufman, T. C. (1984). Defects in embryogenesis in mutants associated with the Antennapedia complex of Drosophila melanogaster. Dev. Biol. 102, 147-172. doi: 10.1016/0012-1606(84)90182-9

Wells, A. R., Zou, R. S., Tulu, U. S., Sokolow, A. C., Crawford, J. M., Edwards, G. S., et al. (2014). Complete canthi removal reveals that forces from the amnioserosa alone are sufficient to drive dorsal closure in Drosophila. Mol. Cell. Biol. 25, 3552-3568. doi: 10.1091/mbc.E14-07-1190

Zeh, D. W., Zeh, J. A., and Smith, R. L. (1989). Ovipositors, amnions and eggshell architecture in the diversification of terrestrial arthropods. Q. Rev. Biol. 64, 147-168. doi: 10.1086/416238

Conflict of Interest Statement: The authors declare that the research was conducted in the absence of any commercial or financial relationships that could be construed as a potential conflict of interest.

Copyright (c) 2015 Horn, Hilbrant and Panfilio. This is an open-access article distributed under the terms of the Creative Commons Attribution License (CC BY). The use, distribution or reproduction in other forums is permitted, provided the original author(s) or licensor are credited and that the original publication in this journal is cited, in accordance with accepted academic practice. No use, distribution or reproduction is permitted which does not comply with these terms. 